\title{
Didactique de l'oral et normes de prononciation : quid du français « standard » dans une approche perceptive ? ${ }^{1}$
}

\author{
Sylvain Detey \& David Le Gac \\ Université de Rouen, E. A. LiDiFra \\ \{sylvain.detey et david.legac\}@univ-rouen.fr
}

\section{Introduction}

La didactique de l'oral, en français langue étrangère (FLE), seconde, mais aussi première, est un domaine $^{2}$ qui peut et doit bénéficier des travaux des linguistes documentant les usages attestés du français parlé. Ces travaux se situent évidemment en amont du champ didactique stricto sensu, mais leurs apports et les interrogations qu'ils peuvent susciter doivent, lorsque cela est possible, être intégrés aux réflexions des méthodologues et des praticiens. Outre les spécificités lexicales, syntaxiques et discursives propres au français parlé, dans ses divers registres et ses diverses variétés, vis-à-vis du français écrit, ce sont bien évidemment la substance (phonétique) et la morphophonologie de ce français parlé qui le caractérisent en premier lieu, assignant ainsi à la «prononciation » et à ses normes une valeur non-négligeable dans l'enseignement/apprentissage de la langue puis dans l'usage qui en est fait, tant en perception qu'en production.

\subsection{Français parlé, normes et représentations langagières}

La multiplication des travaux descriptifs portant sur le français parlé, depuis la constitution du Français Fondamental (Rivenc, 2000), est à cet égard salutaire, car les enseignants, et à leur suite les apprenants, ont besoin d'informations et de données autres que les seules voix et intuition du locuteur-enseignant pour en appréhender les particularités et les mécanismes. En ce sens, la constitution de grands corpus oraux numérisés, qu'ils soient à vocation didactique ou linguistique (Kawaguchi, Zaima \& Takagaki, 2006), est une avancée considérable pour la didactique de l'oral. Les données qu'ils fournissent sont non seulement «authentiquement» orales, mais aussi, pour certains d'entre eux, suffisamment diversifiées pour témoigner de la grande diversité des français parlés dans le monde francophone contemporain. Ceci est particulièrement vrai du corpus PFC (Phonologie du Français Contemporain : usages, variétés et structure - www.projet-pfc.net - Durand, Laks \& Lyche, 2002, 2005), qui compte près de 600 heures d'enregistrements de locuteurs et locutrices natifs de France, de Belgique, de Suisse, du Canada, du Burkina Faso, etc., et qui a conduit au lancement d'un projet d'exploitation didactique de ces données pour l'enseignement du français, le projet PFC-EF (http://www.projet-pfc.net/?pfc-ef) (Detey, Durand, Laks, Lyche \& Nouveau, 2007) ${ }^{3}$. Disposer à la base d'une telle richesse de données est un atout indéniable lorsque l'on souhaite constituer du matériel pédagogique (Delamotte-Legrand \& Penloup, 2007 ; Boutin, Brou-Diallo, Kouadio N'Guessan et Nebout-Arkhurst, 2007). Néanmoins, cette abondance oblige également à réactualiser la sempiternelle question du «Quoi enseigner », plaçant en regard données variées et principes de sélection des contenus. Rappelons alors les quatre principes d'élaboration des normes pédagogiques formulés par Albert Valdman $(1989: 21):$ «1) Elles devraient refléter la parole réelle de locuteurs natifs dans des situations de communication authentiques. 2) Elles devraient se conformer à la vision idéalisée qu'ont les locuteurs natifs de leur propre utilisation de la langue. 3) Elles devraient se conformer aux attentes des locuteurs natifs et à celles des apprenants étrangers concernant le type de comportement linguistique approprié pour des apprenants étrangers. 4) Elles devraient prendre en compte les facteurs de traitement et d'apprentissage $»^{4}$. 
Si de nombreux travaux se sont penchés sur les premier et quatrième de ces principes, relativement peu d'études se sont en revanche consacrées, à notre connaissance, aux deuxième et troisième principes, qui renvoient notamment tous deux aux représentations langagières des locuteurs et des apprenants de la langue cible. En ce qui concerne la didactique de l'oral et les normes de prononciation en français, ces deux principes conduisent aux interrogations suivantes : a) Quelle est la vision idéalisée qu'ont les locuteurs natifs francophones de leur propre prononciation et de celle des autres natifs ? b) Quelles sont les attentes des locuteurs natifs et celles des apprenants étrangers concernant la/les norme(s) de prononciation appropriée(s) pour des apprenants étrangers?

Or, lorsque l'on interroge à brûle-pourpoint des apprenants et des enseignants de français sur le type de français qu'ils souhaitent apprendre ou enseigner, la notion de « français standard » (dorénavant «FS ») émerge généralement assez rapidement. En outre, lorsque l'on consulte certains ouvrages, pédagogiques ou spécialisés, on observe également de nombreuses références à cette notion, parfois superposée à celle de «norme orthoépique » ou encore de français « de référence » (Francard, Géron \& Wilmet, 2001).

Il semble donc admis de la part des apprenants et des enseignants en question que leur vision idéalisée et leurs attentes vis-à-vis du français à apprendre et à enseigner correspondent à la notion de français standard ou à une notion similaire. Partant, le FS serait le français que tout locuteur natif pourrait reconnaître et attendre de la part des étrangers parlant français.

Cependant, la notion même de français standard, ou l'une des notions affiliées, est fondamentalement problématique. Comme le rappelle Laks (2002), la définition du «bon usage», en termes de prononciation, n'est pas aussi aisée à déterminer que celle du bon usage grammatical, syntaxique notamment, lequel se laisse bien plus facilement appréhender dans un ensemble, non clos mais bien circonscriptible, d'écrits de référence. On réalise alors qu'on doit se demander à quoi correspond réellement le français standard. Avant de poursuivre, précisons tout de suite que nous ne traitons ici que du français standard de France ${ }^{5}$ et que nous nous limitons à la composante phonético-phonologique.

Pour cela, nous nous fonderons initialement sur l'excellente synthèse de Morin $(2002)^{6}$ qui expose l'évolution des concepts en question. A travers un examen diachronique des travaux et des discours relatifs aux normes de prononciation (dictionnaires, traités correctifs, manuels ou ouvrages descriptifs ; Thurot, Rousselot, Grammont, Pichon, Bruneau, Fouché, Passy, Malécot, Delattre, Martinet, Walter, Warnant, etc.), Morin décrit plusieurs étapes, parmi lesquelles : 1) le « français de la conversation soignée des milieux parisiens de l'aristocratie, puis de la bonne bourgeoisie » (Pichon, 1938) - notons que c'était ce français qui était inscrit dans les traités et dictionnaires destinés aux étrangers ; 2) le « français parisien de la bourgeoisie cultivée » (Fouché, 1936) puis simplement 3) le « français parisien cultivé » (Fouché, 1956) et 4) le français « de la conversation sérieuse mais détendue de la classe dirigeante de la capitale » (Malécot, 1977). Paris est donc le lieu d'ancrage, et cela même chez les auteurs provinciaux, comme Rousselot (1911-1914), lui-même Angoumoisin (Charente) : "Quoi qu'on puisse en dire et quelles que soient les prétentions de plusieurs villes de France, une seule est le berceau du français : c'est Paris. L'influence de Paris n'est donc pas usurpée ; et c'est à sa langue qu'il faut se conformer ».

Mais, comme l'explique Morin, la suprématie parisienne n'est pas du goût de tous : Bruneau (originaire des Ardennes) écrit en 1931 que la norme est celle du «bourgeois parisien cultivé [...et] plus généralement [du] Français cultivé de toutes les grandes villes du nord de la France » (en italiques dans le texte original) ; Martinon indique, en 1913 : «Pour que la prononciation de Paris soit tenue pour bonne, il faut qu'elle soit adoptée au moins par une grande partie de la France du Nord»; Martinet enfin (originaire de Savoie), en défendant le français des « Parisiens de province », écrivait en 1990: «que ce qui paraissait conditionner l'évolution [de la norme] [...] était le consensus des Français non-méridionaux qui se confondait avec celui de résidence parisienne [... et l'appartenance aux] classes cultivées » et cela correspondait à «une prononciation [...] qui passe inaperçue parce qu'aucun de ses traits ne retient l'attention et détourne la compréhension du message ».

Par la suite, deux nouvelles appellations voient le jour : 1) le français standard, proposé par Léon dans les années 60, "grosso modo représenté par les annonceurs et les interviewers de la radio », mais masquant une certaine réalité sociologique et autorisant toujours une certaine hiérarchisation régionale. Morin note 
à son propos : «Le terme 'français standard', ou plutôt, 'Standard French', est vite devenu à l'étranger un terme relativement creux qui renvoie indifféremment à toutes sortes de variétés de français non méridional». 2) le français standardisé, défini par Carton, Rossi Autesserre et Léon en 1983 de la manière suivante : " Il semble maintenant qu'il existe, dans toutes les régions de France - et non plus seulement à Paris -, une prononciation commune acceptée partout et qu'on pourrait appeler standardisée. On la définira d'abord linguistiquement, par des caractères communs, opposés aux variantes qui excluent leurs auteurs du français standardisé et qui marquent un accent régional » (voir Borrell \& Billières, 1989).

En guise de conclusion à son examen des normes de prononciation, Morin distingue deux conceptions du français de référence, en reprenant les termes des auteurs dont il a examiné les travaux : la première, présentée, à juste titre, comme relativement tautologique par l'auteur, le conçoit comme «celui qui est 'parlé sans accent particulier', ou dont la 'prononciation [...] passe inaperçue', ou encore dont la 'prononciation commune acceptée partout, [est définie] par des caractères communs, opposés aux variantes qui [...] marquent un accent régional' ». La seconde envisage le français de référence comme « celui d'une certaine classe sociale lorsqu'elle adopte un style approprié : d'abord la Cour du roi, puis la haute bourgeoisie parisienne, puis les 'Parisiens authentiques (de naissance, d'éducation, etc.), parmi les chefs d'entreprise, hauts fonctionnaires, cadres supérieurs et tous ceux qui ont des situations de responsabilités dans les professions libérales' ».

Laks (2002), quant à lui, en fait le commentaire suivant: "Ce que Morin appelle le 'français de référence' est, ainsi qu'il le note in fine, une construction purement doxique cumulant descriptions anciennes et analyses classiques, intuitions et remarques personnelles de nombreux phonologues, notations plus contemporaines et analyses partielles d'usages spécifiques ».

Aussi, au vu de l'exposé de Morin et du commentaire de Laks, on est finalement en droit de se demander si la notion même de FS ou toute autre notion assimilée possède une quelconque réalité, sous une forme ou sous une autre, notamment en termes de représentation, dans l'esprit des locuteurs natifs, voire des apprenants, autre que purement « doxique ». C'est bien là la question centrale qui sous-tend cet article et qui rejoint le deuxième principe de Valdman : les locuteurs natifs ont-ils une "vision idéalisée » de leur prononciation, et si oui quelle est-elle? Les enjeux didactiques d'un tel questionnement nous semblent clairs : si, comme nous l'avons évoqué plus haut, les apprenants et les enseignants « réclament » du FS, il convient, pour répondre à leur attente, de vérifier que la notion même de FS est viable. Pour s'en assurer, il faut répondre à deux questions corollaires : qui parle ce FS ? Et comment déterminer son existence ?

\subsection{Quels locuteurs et quelle procédure?}

Lorsque l'on examine les travaux qui pourraient éventuellement nous apporter quelques éléments de réponse, on s'aperçoit qu'ils sont de deux types : d'une part des descriptions phonético-phonologiques des productions de locuteurs de différentes régions de France (par exemple Martinet et Walter, 1973); d'autre part des travaux d'identification perceptive d'accents, régionaux ou étrangers (Boula de Mareüil, Vieru-Dimulescu, Woehrling \& Adda-Decker, 2007). Si l'approche perceptive de ces derniers nous semble indispensable, dès lors que l'on souhaite interroger les représentations, conscientes ou non, des auditeurs, les résultats obtenus ne permettent cependant pas d'interroger la norme à laquelle ils renvoient implicitement (Paris étant présupposé comme point de référence).

Au vu des définitions, parfois très imprécises, des français standard et standardisé, on est par ailleurs en droit de s'interroger sur le choix des locuteurs qui pourront éventuellement servir de locuteurs «de référence ». Des locuteurs parisiens ? Des professionnels de la parole publique ? Autre piste à suivre, dans une optique didactique : celle d'enseignants universitaires français, formateurs de (futurs) professeurs de français (en France et à l'étranger), jugés «sans accent» par leur entourage proche, de classe sociale « moyenne » et assez jeunes pour appartenir à la génération du français dit standardisé. Egalement motivé par des raisons pratiques, notre choix a donc porté sur deux enseignants de profil différent :

- L'un (dorénavant DL), originaire de la région parisienne, pouvant a priori incarner le français standard. Agé de 33 ans au moment de notre étude, il habite à Rouen (Normandie) depuis 5 ans, après 
avoir effectué ses études supérieures à Paris, et passé toute son enfance à Melun (Île de France). Il est donc fortement ancré dans la communauté linguistique d'île de France.

- L'autre (dorénavant SD), originaire du sud de la France mais avec une forte mobilité géographique, pouvant à ce titre représenter le français dit «standardisé » (cf. supra). Agé de 29 ans au moment de notre étude, il habite Rouen depuis 2 ans, après avoir vécu plusieurs années à l'étranger, effectué ses études à Toulouse (Sud-ouest), passé quelques années à Paris au moment de l'adolescence et vécu avant cela dans le Sud-est et le Sud durant toute son enfance. Il a donc pour caractéristique de ne pas être ancré dans une communauté linguistique particulière.

Il s'agissait ainsi pour nous de savoir si ces deux locuteurs pouvaient effectivement être perçus comme parlant un français «standard ", ou, en tout cas, s'en approchant en termes de "distance perceptive » pour des auditeurs natifs de l'Hexagone, et, ce faisant, d'entériner l'existence de l'objet «français standard ». La seule procédure envisageable, à cette fin, était de réaliser un test perceptif, dans lequel un échantillon suffisant d'auditeurs francophones natifs évaluerait les productions de ces locuteurs.

Afin de mettre en place un protocole suffisamment « léger », notamment en termes de durée de passation, pour les auditeurs, nous avons laissé de côté un certain nombre de questions envisageables liées aux jugements épilinguistiques (au sens sociolinguistique du terme) des auditeurs, et nous nous sommes concentrés sur les deux questions suivantes, dont la formulation et l'ordre étaient bien évidemment déterminants :

- Question 1: Dans quelle mesure (sur une échelle de 1 à 6 ) la prononciation des ces phrases/mots présente-t-elle une différence par rapport à votre propre prononciation?

- Question 2: Dans quelle mesure (sur une échelle de 1 à 6) la prononciation de ces phrases/mots estelle proche de la prononciation standard du français?

La question 1 interrogeait donc le degré de différence de prononciation, réelle ou supposée, par rapport à celle de l'auditeur, tandis que la question 2 interrogeait le degré de proximité, réelle ou supposée, par rapport au « français standard », tel que conçu par les auditeurs.

Si la question 2 est bien évidemment celle qui semble aborder directement notre problématique, la question 1 était nécessaire pour deux raisons :

1) Il s'agissait d'abord d'une question contrôle, pour tester la capacité des auditeurs à discriminer plusieurs prononciations.

2) Ensuite, cette première question nous renseignait sur les représentations qu'avaient les auditeurs de leur propre prononciation (vis-à-vis de celles d'autres locuteurs), nous renvoyant ainsi au deuxième principe de Valdman, et nous renseignant, par croisement avec la deuxième question, sur leur positionnement personnel, réel ou supposé, vis-à-vis du «standard ».

Dans la section suivante, nous détaillons l'expérience que nous avons réalisée, avant d'en présenter les résultats.

\section{Description détaillée de l'expérience}

\subsection{Les locuteurs « contrôles »}

Outre les deux locuteurs-tests (DL \& SD), trois autres personnes ont été sélectionnées dans la base PFC (Durand, Laks \& Lyche, 2005). Comme DL et SD, ces trois locuteurs étaient des hommes, d'une classe d'âge similaire (de 25 à 39 ans). La qualité audio des enregistrements était bien évidemment un critère de sélection essentiel et, étant très hétérogène d'un point d'enquête à l'autre, elle a considérablement réduit le nombre de locuteurs potentiels. La dernière variable qui entrait en jeu dans cette sélection était celle de l'accent régional : deux locuteurs ont été choisis avec un accent, le troisième sans accent. Pour cela, nous avons procédé à une expertise auditive de leurs enregistrements, et avons utilisé des logiciels (PRAAT, 
SoundForge) pour visualiser et mesurer les indices acoustiques pertinents de façon objective. Nous avons ainsi veillé à ce que les locuteurs présentent bien des caractéristiques phonétiques déviantes par rapport à ce qui est habituellement décrit comme standard dans les manuels de prononciation et propres à leur zone géographique (par exemple les spécificités phoniques d'un accent du sud), ou, au contraire, diffèrent le moins possible dudit standard pour le locuteur considéré sans accent.

Ces trois locuteurs ont une double fonction. Ils revêtent tout d'abord une fonction de locuteurs « contrôles », en lien avec la question 1 de notre test: des évaluations différentes des locuteurs avec accent et sans accent indiquent que les auditeurs ne sont pas «sourds ». Ensuite, et surtout, - et cela rend compte de leur deuxième fonction - seules de telles différences permettent de savoir s'il existe bel et bien une représentation du FS chez les francophones : la notion de FS n'a de sens que si des locuteurs parlent « d'autres » français, et s'ils sont perçus comme tels. Autrement dit, pour valider notre hypothèse centrale selon laquelle il existe une représentation du FS chez les natifs francophones, il est nécessaire que des locuteurs ayant objectivement un «accent» soient évalués comme moins proches de ce standard que les autres. Au final, on aura compris que c'est l'intrication des deux questions, avec la présence de locuteurs avec et sans accent, qui nous permettra de savoir s'il existe ou non une représentation d'un FS dans l'esprit des francophones natifs. On ne pourra conclure à l'existence d'une telle représentation que si les évaluations entre locuteurs-tests et locuteurs avec accent sont à la fois différentes dans la question 1 et dans la question 2. On espère en outre que les locuteurs-tests seront évalués comme proches du standard. A l'inverse, l'existence d'un FS serait franchement remise en cause s'il n'y avait pas de différence d'évaluation dans la question 2 alors qu'il y aurait des différences dans la question 1, différences garantissant que les auditeurs ne sont pas « sourds».

Nous avons ainsi sélectionné les trois locuteurs-contrôles suivants :

1) Un locuteur de Paris centre-ville «PAR ». Ce locuteur avait entre 25 et 27 ans lors des enregistrements. Il est issu d'une famille aisée mais pas de la haute bourgeoisie, ni de l'aristocratie (Østby, 2007). Il est né et a vécu à Paris $\left(6^{\mathrm{e}}\right.$ arr.) et a fait ses études primaires et secondaires dans un établissement privé du $16^{\mathrm{e}}$ arrondissement, puis a effectué des études supérieures ${ }^{7}$. L'écoute ainsi que l'analyse phonétique ont montré que ce locuteur ne présente pas d'accent et est très proche de ce qui est décrit dans les ouvrages comme locuteur du FS.

2) Un locuteur de Biarritz «BIA » avec un accent. Il avait 31 ans lors des enregistrements; il est né, a été scolarisé et est resté dans le sud de la France toute sa vie (entre St Jean pied de Port, Bayonne, Bordeaux, Toulouse) (Aurnague \& Durand, 2003). Il a un CAPES de mathématique ; il parle basque comme seconde langue.

3) Un locuteur de La Réunion «REU » ${ }^{8}$ de 39 ans, représentant le deuxième locuteur avec accent. Ce locuteur est né et a toujours vécu sur l'île à l'l̂let à Cordes (Bordal \& Ledegen, 2007). Il a suivi des études secondaires et est agriculteur. Sa langue maternelle est le créole mais pratique très régulièrement le français depuis son enfance.

\subsection{Les stimuli et les deux tests de perception}

Notre expérience consistait en fait en deux tests de perception. Ces tests reposaient sur les mêmes locuteurs, les mêmes auditeurs, et les mêmes questions, et se déroulaient suivant la même procédure. Seul le type de stimuli changeait : extraits de texte lu et de conversation dans le premier test (« Test $1 »)$, mots prononcés en isolation dans le deuxième («Test $2 »)$.

La fonction du test 1 était d'obtenir une évaluation globale de la prononciation des locuteurs dans deux types de parole (texte lu et conversation guidée). Le test 2, quant à lui, permettait de recueillir des évaluations sur des phénomènes phonétiques bien circonscrits (cf. description du test 2) : l'objectif était d'avoir une toute première idée de certaines caractéristiques phonétiques du FS. 


\subsubsection{TEST 1 (Texte/Conversation )}

Le test 1 consistait, pour un locuteur, en trois extraits du texte de référence du protocole PFC («Le premier ministre ira-t-il à Beaulieu? »), et en trois extraits de conversation guidée, toujours selon le protocole PFC (cf. Durand, Laks, Lyche, 2002, 2005).

Les trois extraits du texte sont les suivants :

1) «Le maire de Beaulieu - Marc Blanc - est en revanche très inquiet. La cote du Premier Ministre ne cesse de baisser depuis les élections. »

2) «La côte escarpée du Mont Saint-Pierre qui mène au village connaît des barrages chaque fois que les opposants de tous les bords manifestent leur colère. »

3) «Il s'est, en désespoir de cause, décidé à écrire au premier ministre pour vérifier si son village était vraiment une étape nécessaire dans la tournée prévue. »

Les phrases du texte sont toutes déclaratives, avec un nombre de mots similaire. Elles ont été réalisées avec environ la même durée pour chaque locuteur, et, donc, à peu près au même débit (la durée moyenne était de 7,6 s). En fait, ces seules caractéristiques - assertion, longueur, durée - retreignaient déjà drastiquement le choix des phrases dans le texte et aucun critère phonétique n'a prévalu.

Quant aux extraits de conversation, nous avons également sélectionné des phrases déclaratives, dont la durée - en secondes - était la plus proche possible de celle des phrases lues (durée moyenne 6,5 s). Les thèmes évoqués étaient les plus neutres possibles : pas de références géographiques, ni de référence à des activités trop marquées (comme l'agriculture de montagne, les « rallye de bridge », le « scoutisme », ...), et bien évidemment, aucune indication de langue ou de dialecte.

\subsubsection{TEST 2 (Mots)}

En ce qui concerne le test 2, les mots étaient choisis dans la liste du protocole PFC, et ce, en fonction de l'évolution de la norme orthoépique. Pour ne pas avoir un nombre trop élevé de stimuli, nous avons procédé comme suit : quand une opposition phonologique tend à être neutralisée, on a sélectionné le mot contenant le phonème qui tend à disparaître. Par exemple, en ce qui concerne l'opposition classique $/ \mathrm{e} / \sim \mathcal{E} /$, laquelle est de moins en moins réalisée, nous avons choisi un mot avec un [ $[\varepsilon]$ final ( «épais ») car c'est le segment qui tend à être remplacé par [e]. Toutefois, dans le cas des segments persistants, on a choisi certains phonèmes qui présentent une réalisation différente selon les régions (exemple : le $[\tilde{\varepsilon}]$ ). Nous avons finalement sélectionné sept mots, contenant en syllabe finale les voyelles /a/ (« ras »: norme

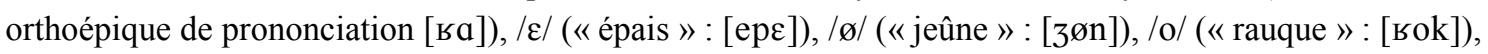

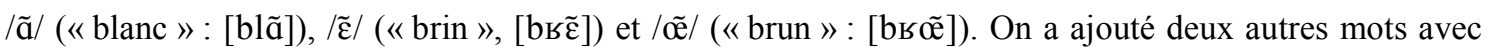

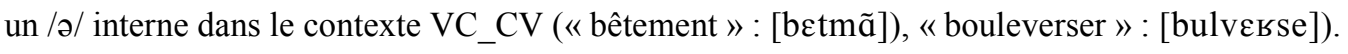

\subsection{Le protocole}

Le test 1 contenait $2 * 3$ extraits $* 5$ locuteurs, i.e. 30 stimuli. Le test 2 contenait 9 mots $* 5$ locuteurs, i.e. 45 stimuli. Pour les deux tests, les stimuli en question ont été mélangés quasi-aléatoirement «à la main »; nous avons simplement évité que deux stimuli d'un même locuteur-test ne se suivent. Au début de chaque test, nous avons ajouté 2 extraits/mots d'entraînement ainsi que 2 extraits/mots en fin de test. Ces stimuli étaient réalisés par 2 locuteurs étrangers aux tests (Brunoy et Lacaune). Les auditeurs devaient donc évaluer 34 stimuli pour le test 1, et 49 pour le test 2, i.e. 83 stimuli pour l'ensemble de l'expérience.

Dans les deux tests, les stimuli étaient séparés par un silence de 6 secondes environ, durant lequel les auditeurs évaluaient le stimulus. Pour le test 2 , chaque mot était répété trois fois de suite, et figurait avec sa représentation orthographique : des mots isolés peuvent être mal compris, et il fallait lever toute ambiguïté dans le cas des paires minimales réelles («brin » « brun »), ou virtuelles (《ras » « rat »).

L'expérience se déroulait dans l'ordre suivant : test 1 , puis test 2 . Les auditeurs devaient donc effectuer un jugement global et devaient ensuite se concentrer sur des particularités phonétiques bien circonscrites. 
L'ordre de passation inverse aurait été problématique : les auditeurs auraient certainement été tentés de se focaliser et de re-chercher les particularités phonétiques en question, et de procéder à un jugement mot à mot, ou presque, au détriment d'une évaluation plus globale.

La tâche des auditeurs était de répondre, pour chaque stimulus, aux deux questions mentionnées dans l'introduction, et que nous présentons en détail ci-dessous. Rappelons que ces deux questions sont identiques pour les deux tests et consistaient à évaluer la prononciation des locuteurs en leur attribuant une note sur une échelle de 1 à 6 :

- Question 1 «Degré de différence de prononciation »: Sur une échelle de 1 à 6 , dans quelle mesure la PRONONCIATION des phrases/mots qui suivent présente-t-elle une différence par rapport à votre propre prononciation? (Echelle : 1) Aucune différence par rapport à votre propre prononciation ; 2) Légère différence ; 3) Différence modérée ; 4) Différence plutôt marquée ; 5) Différence marquée ; 6) Différence très marquée)

- Question 2 « Degré de proximité du français standard»: Sur une échelle de 1 à 6, dans quelle mesure la PRONONCIATION des phrases/mots qui suivent est-elle proche de la prononciation standard du français ? (Echelle : 1) Pas du tout proche du français standard ; 2) Très peu proche ; 3) Assez peu proche ; 4) Assez proche ; 5) Plutôt proche ; 6) Identique)

Au tout début de l'expérience, une remarque expliquait aux auditeurs qu'il s'agissait bien d'évaluer la prononciation et non le «style » ou le « registre » (familier, formel, vulgaire, lu, etc.) de parole.

L'expérience se déroulait sur un site internet, consultable à l'adresse suivante: http://www.projetpfc.net/experiences. Une page d'ouverture présentait l'expérience avec les deux questions et les consignes à respecter. A ce propos, il est à noter que les auditeurs pouvaient écouter les stimuli autant de fois qu'ils le souhaitaient, mais une fois un stimulus évalué, ils avaient pour consigne de ne pas revenir en arrière. A la page suivante, les auditeurs étaient invités à remplir un questionnaire anonyme demandant leur âge, sexe, langue maternelle, lieu de scolarité et lieu de résidence, et s'ils étaient linguistes ou non. Débutait ensuite l'expérience à proprement parler.

En ce qui concerne les auditeurs, nous voulions qu'ils soient de lieux de scolarité et de résidence assez variés pour avoir des résultats exploitables à la question 1 . Nous avons donc sollicité nos réseaux de connaissances dans différentes régions, en les invitant à se connecter au site. Ils pouvaient également faire suivre l'annonce à des personnes « sérieuses » de leur entourage.

Nous sommes conscients des limites de cette procédure : nous n'avions aucun contrôle sur les auditeurs, et ils n'ont pas passé l'expérience dans des conditions de laboratoire. Mais c'était le seul moyen pour nous d'obtenir, assez rapidement, un nombre suffisant de sujets d'origines variées.

Au final, 58 auditeurs ont passé le test 1, et 56 , le test $2: 2$ locuteurs ont abandonné en cours de passation. L'âge des locuteurs varie de 21 à 74 ans et se répartit de la manière suivante : 21-30 ans : 21 sujets ; 31$40: 13 ; 41-50: 7 ; 51-60: 6 ; 61-70: 9$; et 71-74:2. 39 femmes et 19 hommes ont passé l'expérience. Il y a eu 42 non-linguistes et 15 linguistes ( 1 non renseigné) ; 54 personnes de langue maternelle française et 4 d'une autre langue.

Pour les lieux de scolarité, 13 auditeurs l'ont passée hors de France (5 Canada, 2 Suisse, 2 Japon, 1 Bahreïn, 1 Madagascar, 1 Île Maurice et 1 « Multinational»), et 45 en France dont 2 à La Réunion, et 1 à la Martinique. Quant aux 42 restants, nous les avons répartis en auditeurs ayant suivi une scolarité dans un département où l'on parle 1$)$ un dialecte d'oïl (28 auditeurs, dont 11 de région parisienne); 2 ) un dialecte provençal ( 8 auditeurs), et 3 ) un autre dialecte d'oc (6 auditeurs). En faisant cette répartition, on cherchait à distinguer les auditeurs de la région de Marseille et ceux de Toulouse.

\section{Résultats et discussion}

Dans le cadre de cet article, nous n'examinerons que les évaluations effectuées par les auditeurs de langues maternelle française. De même, nous n'allons pas prendre en compte l'effet du sexe, de l'âge, et de l'expertise ( \pm linguiste). Quant à l'impact des lieux de résidence et de scolarité, nous ne retiendrons 
dans notre analyse que celui du lieu de scolarité, lequel nous semble plus important et avoir plus d'effet sur les évaluations. Ceci étant, très peu d'auditeurs ont des lieux de résidence et de scolarité dans des zones dialectales différentes (5 en tout).

\subsection{Résultats du Test 1 (extraits du texte et de conversation)}

\subsubsection{Question 1 (" Degré de différence de prononciation »)}

Les résultats de la question 1 du test 1 sont représentés dans la figure 1. Celle-ci montre clairement des différences d'évaluation entre trois groupes de locuteurs : 1) celui de DL (moyenne globale de 2,24), SD $(2,70)$ et PAR $(2,49), 2)$ celui du locuteur BIA $(3,77)$, et 3$)$ celui du locuteur REU $(4,75)$. Ces différences d'évaluation nous assurent clairement que les auditeurs dans leur ensemble ne sont pas "sourds », et que le protocole expérimental est opératoire.

En outre, si l'on regarde maintenant de plus près les résultats en fonction du lieu de scolarité des auditeurs, on s'aperçoit que ces derniers ont fait des évaluations cohérentes et convergentes. Cela apparaît notamment à travers : 1) les résultats du locuteur REU, dont la prononciation a été évaluée comme très différente de celle des auditeurs, quel que soit leur lieu de scolarité, et 2) les évaluations des auditeurs « oill », qui jugent la différence de prononciation du locuteur BIA comme étant plutôt différente de façon marquée $(3,81)$ alors qu'ils évaluent la prononciation des locuteurs DL, SD et PAR comme présentant une différence légère et modérée par rapport à la leur.

Si l'ensemble des résultats correspond globalement à nos attentes, on remarquera néanmoins que les auditeurs « oc » ne jugent pas comme très différente de la leur les prononciations de DL, SD et PAR alors qu'on aurait pu s'attendre à des résultats comparables à ceux des auditeurs "prov » pour ces trois locuteurs. Autre point d'interrogation, les évaluations des auditeurs «prov» à propos de $\operatorname{SD}(3,64)$ comparées à celles de DL, PAR et BIA : on se serait en effet attendu à ce que SD obtienne une moyenne similaire à celle de DL ou PAR, ou, en tout cas, inférieure à celle de BIA.

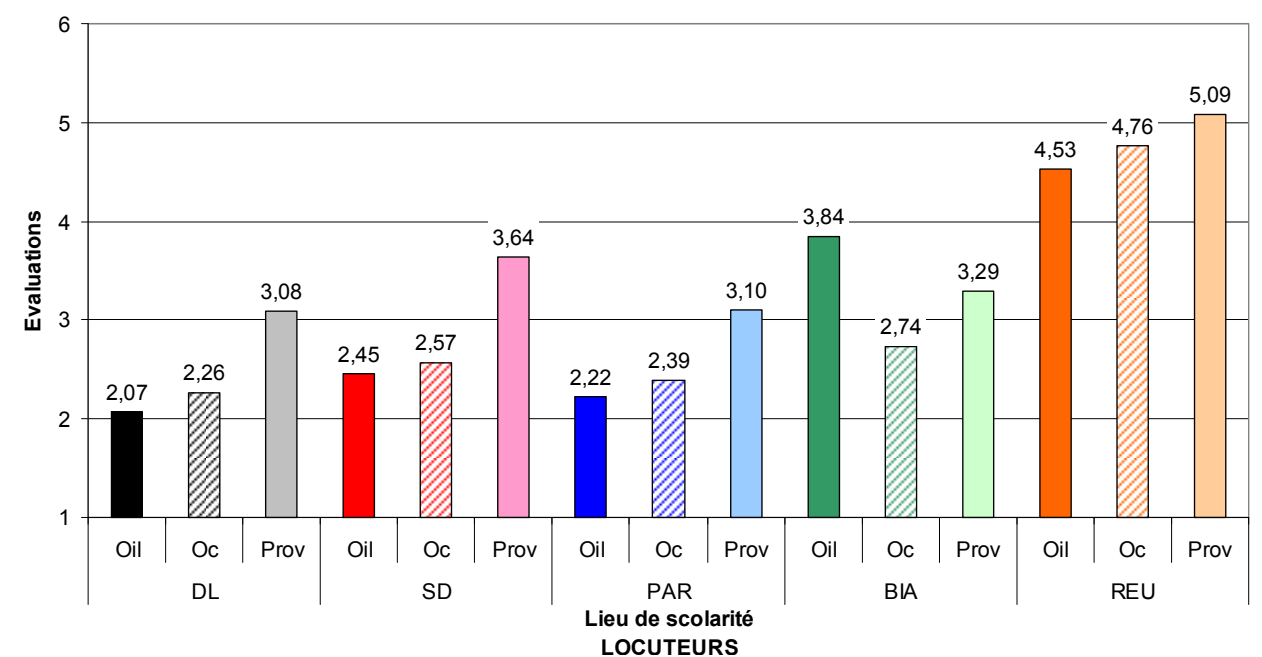

Figure 1. Résultats du test 1 pour la question 1 (degré de différence de prononciation) pour chaque locuteur en fonction du lieu de scolarité des auditeurs

\subsubsection{Question 2 ("Degré de proximité du français standard »)}

Les résultats de la question 2 du test 1 sont représentés dans les figures 2 et 3 . Ces résultats ont été soumis à une analyse de variance (ANOVA) qui a révélé un effet de la variable locuteur (sans distinction des types de parole - figure 2) sur les évaluations $(\mathrm{F}(4,1646)=204,421 ; \mathrm{p}<0.0001)$. Dans le détail, des tests-t 
comparant deux à deux les locuteurs ont montré que : 1) DL a été jugé comme significativement différent des autres locuteurs et proche du FS $\left(\right.$ Moy $\left._{D L}=5,031, \mathrm{p}<0.001\right) ; 2$ ) les locuteurs SD et PAR ne se différencient pas, et forment une catégorie plutôt proche du FS $\left(\right.$ Moy $_{\mathrm{SD}}=4,686$; Moy $_{\mathrm{PAR}}=4,601$; $\mathrm{p}=0.3612)$; 3) les locuteurs BIA et REU sont tous les deux significativement différents à la fois l'un de l'autre et de tous les autres locuteurs ; on remarquera que le locuteur REU est celui qui a été jugé comme le moins proche du FS.

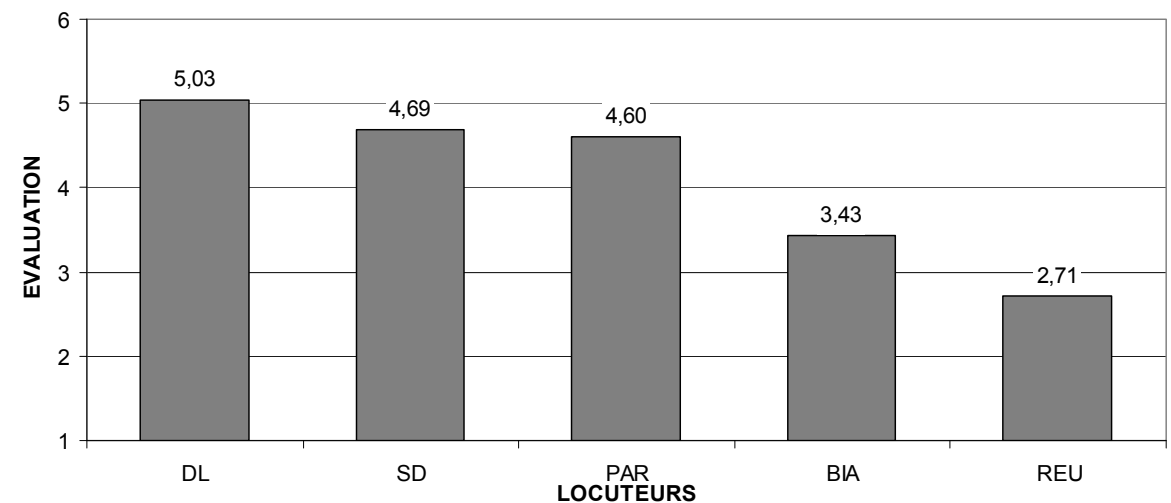

Figure 2. Résultats du test 1 pour la question 2 (proximité du français standard) pour chaque locuteur.

Ces résultats nous permettent de répondre globalement aux principales questions de cet article. Tout d'abord, ils confirment que DL et SD sont perçus comme des locuteurs plutôt proches du FS. Ensuite, et surtout, le fait qu'il y ait des différences significatives entre les évaluations des locuteurs et que celles-ci soient cohérentes (i.e. le fait que SD, DL et PAR soient proches du FS tandis que BIA et surtout REU soient évalués comme plutôt éloignés) suggère que le FS possède effectivement une existence linguistique dans l'esprit des auditeurs natifs du français.

Cependant, on remarque également que, contrairement à notre hypothèse initiale, SD et DL ne sont pas évalués de la même manière : DL est considéré plus proche du FS que SD. Dans un premier temps, on pourrait attribuer cette différence à l'influence de leurs profils sociolinguistiques, mais l'absence de différence entre PAR et SD suggère plutôt un effet du type de parole, ce que confirment les résultats en figure 3 présentant les évaluations pour les extraits de texte et de conversation.

En effet, l'élément notable dans cette figure réside dans la différence significative entre les évaluations du texte et de la conversation du locuteur PAR : la lecture du texte est jugée comme beaucoup plus proche du FS que la conversation, ce qui s'explique probablement en partie par son style plus "relâché » dans la conversation (lexique, marqueurs discursifs - i.e. «ben », « quoi ») - cela fait aussi écho à la « conversation sérieuse mais détendue » de Malécot (1977) citée par Morin (2002).

Aussi, lorsque, en écoutant les enregistrements, on réalise que le style de SD dans la conversation est beaucoup moins « relâché » que celui de PAR, on comprend pourquoi SD est jugé plus proche du FS que PAR en conversation, alors que c'est l'inverse qui se produit en lecture (ce qui rejoint le classement général plaçant DL et PAR comme plus proche du FS).

Ajoutons finalement qu'on retrouve une différence significative entre la conversation et le texte chez le locuteur BIA, ce qui pourrait s'expliquer soit de la même manière que pour le locuteur PAR, soit par une influence de la tâche de lecture et du facteur orthographique (Chevrot et Malderez, 1999 ; Detey, 2005 ; Detey, Durand \& Nespoulous, 2005). 


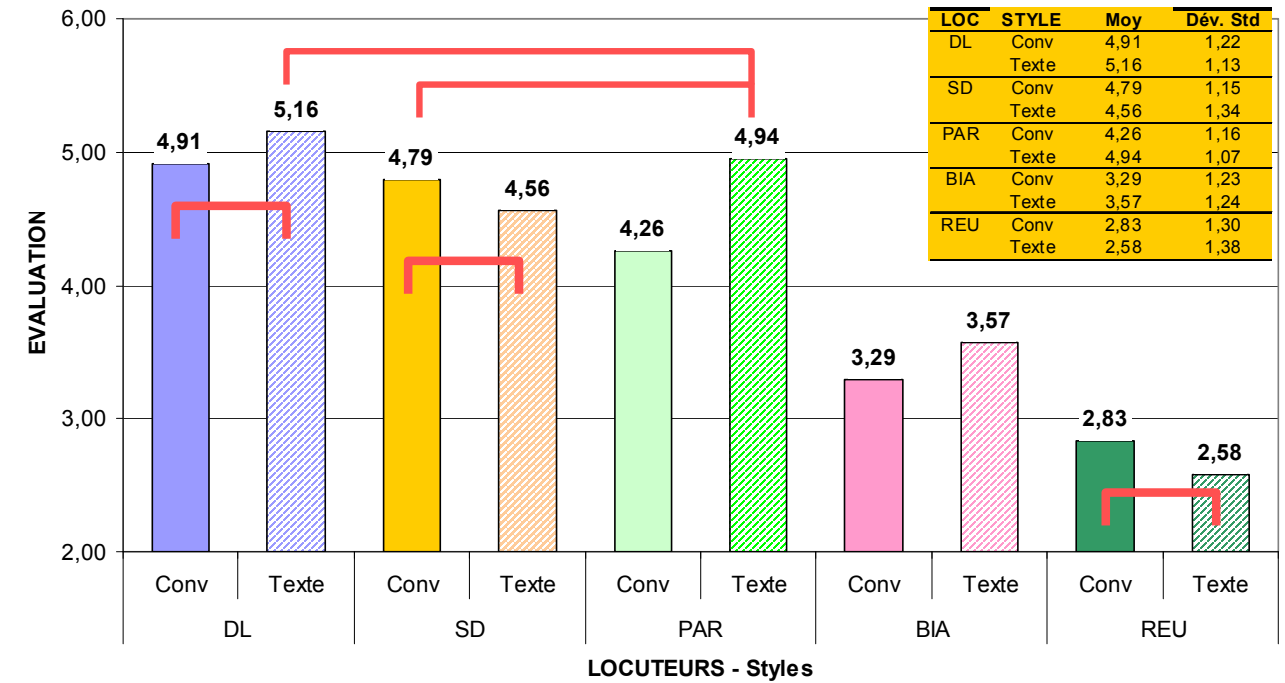

Figure 3. Résultats du test 1 pour la question 2 (proximité du français standard) pour chaque locuteur en fonction du style de parole (texte et conversation). Les lignes reliant deux barres d'histogramme signalent une différence $N O N$ significative

\subsection{Résultats du Test 2 (Mots)}

Nous ne présentons ci-dessous dans la figure 4 que les résultats de la question 2 pour le test 2. L'analyse des résultats mot à mot offre une porte d'entrée vers une définition plus précise des caractéristiques phonético-phonologiques du FS, mais cela demanderait davantage d'analyses. Nous nous contenterons ici d'exposer les résultats globaux obtenus.

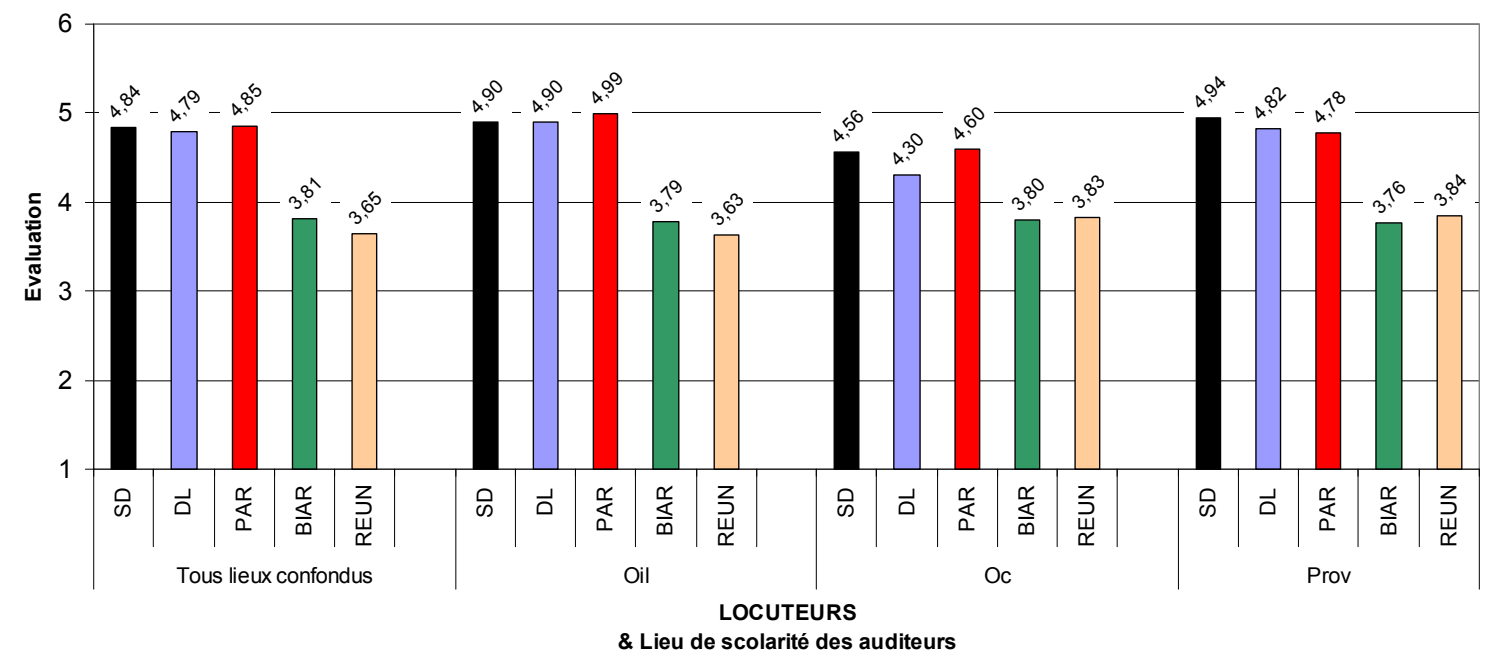

Figure 4. Résultats du test 2 pour la question 2 (proximité du français standard) pour chaque locuteur en fonction du lieu de scolarité.

Ces résultats globaux (moyenne des évaluations pour tous les mots) confirment les tendances observées jusqu'ici. On remarque encore une fois des différences d'évaluation entre les locuteurs, mais de manière beaucoup moins nuancée. Ne ressortent ici que deux groupes de locuteurs au lieu des quatre mis au jour précédemment, avec néanmoins des différences très nettes : d'une part, un groupe formé par SD, DL et PAR, d'autre part, un groupe formé par BIA et REU, qui ne sont plus différenciés dans ce test. On notera 
aussi que, dans ce test-ci, DL est évalué comme légèrement moins proche du FS que SD et PAR, contrairement aux résultats du test 1 .

Les résultats du test 2 montrent donc que les auditeurs sont à même de réaliser une évaluation sur des mots pris hors contexte, mais le nivellement observé dans les évaluations laisse toutefois penser que les facteurs prosodiques et le style de parole, davantage perceptibles en parole continue, jouent un rôle essentiel dans la perception et la représentation du français standard.

\section{Conclusion}

Notre étude a montré que les locuteurs-auditeurs natifs de l'Hexagone étaient en mesure d'évaluer des productions orales comme "plus ou moins standard», en termes de prononciation, et que leurs évaluations étaient relativement convergentes. Ces résultats nous ont permis d'affirmer que la notion de français standard n'était pas une simple construction doxique, mais qu'elle existait au niveau des représentations linguistiques dans l'esprit des locuteurs-auditeurs natifs. D'un point de vue didactique, on pourra donc, tout en encourageant l'exposition à la variation en réception, répondre aux attentes des apprenants et enseignants qui souhaitent prendre comme modèle de production une prononciation plutôt « standard» du français. Cependant, d'autres études restent nécessaires pour pouvoir précisément définir les caractéristiques phonético-phonologiques de ce français standard, et il faut, en outre, dépasser le cadre hexagonal auquel nous nous sommes limités dans notre étude pour prendre en compte le statut des autres « standards » nationaux (belge, suisse, etc.). Alors que la sensibilisation des apprenants de français à la variation langagière fait l'objet d'une attention particulière de la part des didacticiens, notamment vis-àvis de la composante sociolinguistique de la compétence à communiquer langagièrement telle que définie dans le Cadre Européen Commun de Référence pour les Langues, mais aussi vis-à-vis de la Francophonie, dans lequel le français, sous toutes ses formes, fait office de langue véhiculaire internationale, la notion de "français standard » mérite donc d'être réexaminée, à l'aune des grands corpus comme PFC d'une part, mais aussi à celle des travaux récents d'identification d'accents étrangers ou régionaux d'autre part. Cette étude, nous l'espérons, y aura contribué.

\section{Références bibliographiques}

Aurnague, M. \& Durand, J. (2003). Quelques aspects de la phonologie du français au Pays basque. La tribune internationale des langues vivantes, 33, 108-116.

Bordal, G. \& Ledegen, G. (2007). Le français de la Réunion: lexique, morphosyntaxe, alternance codique et prononciation. In Detey, S. \& Nouveau, D. (éds.). Bulletin PFC, 7, 121-133.

Borrell, A. \& Billières, M. (1989). L'évolution de la norme phonétique en français contemporain. La Linguistique, 25, fasc. 2, 45-62.

Bouchard, R. (2003). Didactiques de l'oral et éducation communicative implicite. Colloque Didactiques de l'oral, Université de Montpellier III et IUFM, 14-15 juin 2002. In Maurer B. (éd.). Les Actes de la DESCO « Didactiques de l'oral ». Caen : CRDP de Basse Normandie.

Boula de Mareüil, P., Vieru-Dimulescu, B., Woehrling, C. \& Adda-Decker, M. (2007). Caractérisation d'accents étrangers et régionaux en français : vers une identification automatique. Colloque international Regards croisés sur la phonologie du français contemporain, Paris, MSH, décembre 2007.

Boutin, A. B., Brou-Diallo, A. C., Kouadio N'Guessan, J. \& Nebout-Arkhurst, P. (2007). PFC-EFLS : de l'intérêt du projet PFC-EF pour la didactique du français, langue seconde en Côte d'Ivoire. In Detey, S. \& Nouveau, D. (éds.). Bulletin PFC, 7, 65-85.

Chanfrault-Duchet, M.-F. (2003). Restaurer l'oralité en classe de français. Colloque Didactiques de l'oral, Université de Montpellier III et IUFM, 14-15 juin 2002. In Maurer B. (éd.). Les Actes de la DESCO « Didactiques de l'oral ». Caen : CRDP de Basse Normandie, 47-57.

Chevrot, J.-P. \& Malderez, I. (1999). L'effet Buben : de la linguistique diachronique à l'approche cognitive (et retour). Langue Française, 124, 104-125. 
Chiss, J.-L. (2003). Le couple oral/écrit et la tension entre communicatif et cognitif. Colloque Didactiques de l'oral, Université de Montpellier III et IUFM, 14-15 juin 2002. In Maurer B. (éd.). Les Actes de la DESCO « Didactiques de l'oral ». Caen : CRDP de Basse Normandie, 13-21.

Delamotte-Legrand, R. \& Penloup, M.-C. (2007). Intérêt et usages des documents PFC en langue première. In Detey, S. \& Nouveau, D. (éds.). Bulletin PFC, 7, 55-64.

Detey, S. (2005). Utiliser l'écrit au service de l'oral. Le français dans le monde, 342, 38-40.

Detey, S., Durand, J., Laks, B. Lyche, C. \& Nouveau, D. (2007). Voix de la francophonie, éducation langagière et corpus numérisé : PFC-EF, des ressources pour la didactique du français. In Detey, S. \& Nouveau, D. (éds.). Bulletin PFC, 7, 11-29.

Detey, S., Durand, J. \& Nespoulous, J.-L. (2005). Interphonologie et représentations orthographiques. Le cas des catégories $/ \mathrm{b} /$ et $/ \mathrm{v} /$ chez des apprenants japonais de Français Langue Etrangère. Revue PArole, 34/35/36 (supplément), 139-186.

Durand, J., Laks, B. \& Lyche, C. (2002). La phonologie du français contemporain : usages, variétés et structure. In Pusch, C. \& Raible, W. (eds.). Romanistische Korpuslinguistik- Korpora und gesprochene Sprache / Romance Corpus Linguistics - Corpora and Spoken Language. Tübigen : Gunter Narr Verlag, 93-106.

Durand, J., Laks, B. \& Lyche, C. (2005). Un corpus numérisé pour la phonologie du français. In Williams, G. (éd.). La linguistique de corpus. Rennes : Presses Universitaires de Rennes, 205-217.

Francard, M., Géron, G. \& Wilmet, R. (éds) (2001). Le français de référence. Constructions et appropriations d'un concept. Louvain-la-Neuve : Peeters.

Kawaguchi, Y., Zaima, S. \& Takagaki, T. (éds.) (2006). Spoken Language Corpus and Linguistic Informatics. Amsterdam/Philadelphia : John Benjamins,

Laks, B. (2002). Description de l'oral et variation : la phonologie et la norme. L'information grammaticale, 94, 5-10.

Martinet, A. \& Walter, H. (1973). Dictionnaire de la prononciation française dans son usage réel. Paris : France Expansion.

Morin, Y.-C. (2000). Le français de référence et les normes de prononciation. Cahiers de l'Institut de linguistique de Louvain, 26 (1), 91-135.

Østby, K. A. (2007). Aspects du français oral : la haute bourgeoisie parisienne. In Detey, S. \& Nouveau, D. (éds.). Bulletin PFC, 7, 227-238.

Plane, S. (2005). L'oral: quelles pratiques pour apprendre à travailler ensemble \& Quel oral enseigner pour favoriser la réussite des élèves en français? Intervention stage PAF, Paris VII, 2 février 2005.

Rivenc, P. (2000). Pour aider à apprendre à communiquer dans une langue étrangère. Paris/Mons : Didier/CIPA.

Valdman, A. (1989). The elaboration of pedagogical norms for second language learners in a conflictual diglossia situation. In Gass, S., Madden, C., Preston, D. \& Selinker, L. (eds.). Variation in Second Language Acquisition, Volume I : Discourse and Pragmatics. Clevedon: Multilingual Matters. 15-34.

Walter, H. (1982). Enquête phonologique et variétés régionales du français. Paris : PUF.

\footnotetext{
${ }^{1}$ Tous nos remerciements à Jacques Durand qui nous a incité à entreprendre cette étude et qui nous a généreusement prodigué ses conseils lors de sa réalisation, ainsi qu'à tous nos collègues, amis et internautes inconnus pour avoir participé à notre expérience. Une partie de ce travail a été présentée lors du colloque international Regards croisés sur la phonologie du français contemporain, tenu en décembre 2007 à Paris. Nous remercions également les deux relecteurs anonymes du CMLF-08.

${ }^{2}$ Pour un panorama du champ, voir par exemple les Actes du séminaire « Didactiques de l'oral » (juin 2002), publiés en 2003 par la Direction générale de l'Enseignement scolaire et accessibles en ligne à l'adresse suivante: http://eduscol.education.fr/D0126/didactiqueoral acte.htm. Consulter en particulier (Chiss, 2003), (Bouchard, 2003), (Chanfrault-Duchet, 2003). Voir également l'intervention de Sylvie Plane (2005) concernant les recherches sur l'oral, dont une synthèse est accessible en ligne à l'adresse suivante: http://www.accreteil.fr/langages/contenu/prat peda/dossiers/oral.htm\#recherches oral
} 
ISBN 978-2-7598-0358-3, Paris, 2008, Institut de Linguistique Française

Didactique et enseignement, français langue maternelle, français langue seconde DOI $10.1051 / \mathrm{cmlf08209}$

${ }^{3}$ Consultable à l'adresse suivante $:$ http://www.projet-pfc.net/?u_s $=4 \& u_{-} \mathrm{a}=157 \&$

${ }^{4}$ Notre traduction.

${ }^{5}$ Les « standards » québécois ou ivoirien, par exemple, peuvent être différents du « standard » français.

${ }^{6}$ A laquelle nous empruntons les citations de cette section et à laquelle nous renvoyons le lecteur pour davantage d'information, ainsi que pour les références précises.

${ }^{7}$ La fiche signalétique de PAR était quasi vide. Son profil a été établi à partir des enregistrements de ses conversations où il se décrit en détail.

${ }^{8}$ Les identifiants des locuteurs dans la base PFC sont respectivement : $75 c c b 2$ pour le locuteur PAR, 64api pour le locuteur BIA, et $974 g r$ pour le locuteur REU. 